\title{
Characterization and Beige Adipogenic Potential of Human Embryo White Adipose Tissue-Derived Stem Cells
}

\author{
Chuanhai Zhang ${ }^{a, b, c}$ Jing Jing Wang ${ }^{c}$ Xiaoyun $\mathrm{He}^{\mathrm{a}, \mathrm{b}}$ Chao Wang ${ }^{\mathrm{c}}$ \\ Boyang Zhang ${ }^{a, b}$ Jia Xu $u^{a, b}$ Wentao Xu $u^{a, b}$ Yunbo Luo ${ }^{a, b}$ Kunlun Huang \\ aBeijing Advanced Innovation Center for Food Nutrition and Human Health, College of Food \\ Science and Nutritional Engineering, China Agricultural University, Beijing, 'Key Laboratory of Safety \\ Assessment of Genetically Modifed Organism (Food Safety), Ministry of Agriculture, Beijing, 'Key \\ Laboratory of Animal Ecology and Conservation Biology, Institute of Zoology, Chinese Academy of \\ Sciences, Beijing, China
}

\section{Key Words}

Embryo white adipose tissue-derived stem cells $\bullet$ Characterization $\bullet$ Beige Adipogenic Potential - Gene feature

\begin{abstract}
Background/Aims: Brown and beige adipocytes are widely recognized as potential therapeutic targets to treat obesity and related metabolic disorders, and the recruitment of brown and beige adipocytes is an essential aspect that requires attention. Although many methods of activating brown adipocytes or generating beige adipocytes have been reported, the limited number and sources are the biggest challenges. The number of white adipocytes is much greater than the number of brown adipocytes, both in human adults and fetuses. Unfortunately, human adult white adipose tissue-derived stem cell (aWAsc) has little beige adipogenic potential. However, the characteristics and beige adipogenic potential of human embryo-derived white adipose stem cells (eWAsc) still need to be investigated. Methods: To analyze the characteristics and functionality of eWAsc, we analyzed the markers of adipose precursor cells by flow cytometry. Then, differentiation and browning/beiging were induced, and the identifying markers were analyzed by real-time PCR and immunoblot. In addition, more in-depth exploration was performed using RNA-SEQ on eWAsc and aWAsc. Results: eWAsc was isolated from human embryonic white adipose tissue, and aWAsc was isolated from adult white adipose tissue by collagenase treatment. eWAsc has extreme advantages in adipogenesis capacity and browning/beiging ability in comparison to aWAsc, indicating that eWAsc may possess some special regulatory factors to promote the generation of functional brown/beige adipocytes. Greater exploration was enabled by RNA-SEQ, revealing a large number of differences at the transcriptional levels, including 1263 differentially expressed genes, 657 down- and 605 upregulated, in eWAsc compared to aWAsc. Pathway analysis revealed enrichment in cell cycle, TGF- $\beta$ signaling pathway, DNA replication, and Hippo

Yunbo Luo

and Kunlun Huang

College of Food Science and Nutritional Engineering, China Agricultural

University, No. 17 Tsinghua Donglu, Beijing 100083 (China)

E-Mail hk1009@163.com; lyb@cau.edu.cn
\end{abstract}


Zhang et al.: Beige Adipocytes and Human Embryo White Adipose Tissue-Derived Stem Cells

signaling pathways. Interestingly, the expression levels of C/EBP $\alpha$, FGF1 and FST gene, which are related to the maturation of adipocytes, Hippo signaling pathway and TGF- $\beta$ signaling pathway, were significantly higher in eWAsc than in aWAsc. These may be potential candidates and possible regulatory targets for recruiting beige adipocytes in human adipose tissue. Conclusion: Overall, we have demonstrated the molecular characteristics and excellent beige adipogenic potential of eWAsc, providing a new reference for studying human adipocytes.

(C) 2018 The Author(s)

Published by S. Karger AG, Basel

\section{Introduction}

There are two main types of adipose tissue: white adipose tissue (WAT) and brown adipose tissue (BAT) [1]. In both of these tissues, the adipocytes have obvious lipid droplets, although the functions of these cells are distinct and different. White adipocytes are mainly responsible for energy storage and contain a single, large lipid droplet, whereas brown adipocytes maintain body temperature and energy consumption through thermogenesis via the activation of uncoupling protein 1 (UCP1) [1, 2]. Brown adipocytes contain multilocular lipid droplets and have abundant mitochondria, which decouple proton transfer across the membrane during ATP synthesis [3]. Interestingly, "beige adipocytes" are specialized cells that function similarly to brown adipocytes and originate from special adipose precursors $[4,5]$. In recent years, there has been a large increase in the occurrence of obesity and related metabolic diseases, including type 2 diabetes, cardiovascular disease, hypertension, and hyperlipidemia [6, 7]. Brown adipocytes and beige adipocytes are widely recognized as potential therapeutic targets to treat obesity and related diseases.

There has been significant progress in revealing and understanding the mechanisms that underlie brown/beige adipocyte development and activation in rodent models, but there has been insufficient research regarding this topic in human adults and fetuses. Importantly, there is limited understanding of the quantity and utilization of both adult and infant BAT. Studies have compared human embryonic brown adipocytes by transcriptomic technology and found marker gene expression with both classical brown adipocytes and beige adipocytes, indicating that human embryo BAT has mixed features of both classic brown and beige adipocytes $[5,8,9]$. In addition, several studies have investigated the molecular properties of primary brown adipocytes derived from human fetal interscapular and perirenal depots, revealing that the brown adipogenic potential of brown fat precursor cells isolated during different stages of fetal development is significantly higher than in adults $[8,9]$. However, the molecular characterization and beige adipogenic potential of primary adipocytes/ stem cells derived from typical human fetal subcutaneous white fat depots (eWAsc) has not been clarified. This subject needs to be investigated to provide a more comprehensive understanding of beige development and recruitment. In order to address this subject, we successfully isolated eWAsc from human fetal fat tissues and investigated the flow cytometry features, beige adipogenic potential, thermogenic capacity, metabolic function, and gene signature (RNA-SEQ) in comparison to aWAsc.

Herein, we describe the beige adipogenic potential and characteristics of human primary white adipocytes from human fetal subcutaneous white fat depots. We successfully cultured these cells and described a comprehensive beige adipocyte phenotype. The regulatory features and mechanisms are discussed at the level of transcriptome analysis. We believe that this information will provide a valuable tool for both validating existing rodent targets and identifying important questions in studying human beige adipocytes. 
Zhang et al.: Beige Adipocytes and Human Embryo White Adipose Tissue-Derived Stem Cells

\section{Materials and Methods}

\section{Human subjects}

Human embryonic white adipose tissue was obtained from spontaneous abortions (11 weeks of gestation) at the Department of Gynecology and Obstetrics in $\mathrm{Lu} \mathrm{He}$ hospital. Subcutaneous adipose tissues were dissected from the leg and back of the embryo. In parallel, adult subcutaneous fat tissues were obtained from a colon cancer patient in the Department of Surgery in Lu He Hospital. Fetal parents and patients were free of cardiovascular disease, endocrine diseases, metabolic disorders, and acute infection. Isolation of stem cells/primary adipocytes from adipose tissues of human embryo and adult subcutaneous fat was performed as described previously [8]. Briefly, immediately after dissection, freshly resected fat depots were collected, minced, and digested using collagenase I ( $2 \mathrm{mg} / \mathrm{mL}$ in PBS with the addition of 3.5\% BSA; Worthington Biochemical Corporation, Lakewood, NJ) and the stromal vascular fraction (SVF) was isolated. Floating adipocytes were separated from the SVF by centrifugation at $300 \times \mathrm{g}$ for $3 \mathrm{~min}$. The study complied with the Helsinki Declaration for investigation of human subjects. It received ethical approval from the competent Institutional Review Boards of the Capital Medical University. All participants provided written informed consent.

\section{Culture of primary human white fat progenitors}

After isolation, SVF cells were cultured in DMEM/Ham's F-12 1:1 (DMEM/F12) containing 20\% (vol/ vol) fetal bovine serum (FBS) (Sigma-Aldrich) and antibiotics (1\% penicillin-streptomycin). For adipocyte differentiation, cells were grown to reach $100 \%$ confluence and then exposed to brown adipogenic induction cocktail in high-glucose Dulbecco's modified Eagle's medium (DMEM/H) containing 0.5 mM isobutylmethylxanthine, $0.1 \mu \mathrm{M}$ dexamethasone, $0.5 \mu \mathrm{M}$ human insulin (Sigma-Aldrich, Dallas, TX), $2 \mathrm{nM}$ T3, $30 \mu \mathrm{M}$ indomethacin, $17 \mu \mathrm{M}$ pantothenate, $33 \mu \mathrm{M}$ biotin, and 2\% FBS for 6-7 days. The induction medium was refreshed every 2 days. To induce eWAC activity, cells were stimulated with $1 \mu \mathrm{M}$ norepinephrine for 4 hours at the end of differentiation. Then, cell samples were collected for RNA extraction or immunoblotting.

\section{Flow cytometry}

The isolated cells from aWAT and eWAT were stained with antibody cocktails that included APCconjugated anti-human CD29 (San Diego, CA, clone TS2/16), FITC-conjugated anti-human CD11b (MiltenyiBiotec, 130-081-201), PE-conjugated anti-human CD31 (MiltenyiBiotec, 130-091-610), and PerCP-Cy5.5-conjugated anti-human CD34 (BDPharmingen). Adipocyte progenitors are defined as CD29+/ CD34+/CD31-/CD11b- cells as analyzed by FACS (BD FACS Aria, BD Biosciences, CA, USA). Data analysis was performed using BD FACS Diva software (BD Biosciences, CA, USA). 


\section{Cellular Physiology Cell Physiol Biochem 2018;51:2900-2915 \begin{tabular}{l|l|l} 
and Biochemistry Published online: 14 December 2018 & $\begin{array}{l}\text { (c) } 2018 \text { The Author(s). Published by S. Karger AG, Basel } \\
\text { www.karger.com/cpb }\end{array}$ \\
\hline
\end{tabular} \\ Zhang et al.: Beige Adipocytes and Human Embryo White Adipose Tissue-Derived Stem Cells}

\section{Real-time PCR}

Total RNA was isolated using Trizol reagents (Invitrogen) following the manufacturer's protocol. RNA quality of the samples was validated by a Nanodrop 2000 (Thermo Scientific, Wilmington, DE, USA). Reverse transcription of total RNA ( $2 \mathrm{ug}$ ) was performed using a high-capacity cDNA reverse transcription kit (Promega Biotech Co., Ltd). Quantitative reverse transcriptase PCR (qRT-PCR) was performed with SYBR Green Master Mix (Promega Biotech Co., Ltd). PCR reactions were run in triplicate for each sample using ABI Prism VIIA7 real-time PCR (Applied Biosystems). Cyclophilin was used as a reference gene, as it is a common and stable internal reference gene which has been used in a previous study [8]. The sequences of primers used in this study are provided in Table 1. In order to guarantee the internal reference we used is a stable and effective internal reference for the sample of human WAsc, we screened and verified 5 pairs of internal reference, and finally determined that the internal reference used in this study is Cyclophilin A (Forward: TGTGTCAGGGTGGTGACTTC, Reverse: GTCTTGGCAGTGCAGATGAA). See the supplementary materials for details.

\section{Immunoblotting}

Total protein was extracted from differentiated adipocytes using RIPA buffer (150 mM sodium chloride, $1.0 \%$ Triton X-100, 0.5\% sodium deoxycholate, $0.1 \%$ SDS, $50 \mathrm{mM}$ Tris, protease and phosphatase inhibitor cocktail (Roche Diagnostics Corp, Pleasanton, CA, USA)). Total protein concentration was measured using a BCA Assay Kit (Pierce Diagnostics Corp, Pleasanton, CA, USA). Equal amount of protein lysates were boiled with $4 \times$ NuPage LDS loading buffer (Invitrogen) and separated by 10\% SDS-PAGE, transferred to a PVDF membrane (Millipore Billerica, MA, USA). After being blocked in 5\% skim milk dissolved in Trisbuffered saline (0.02 M Tris base, $0.14 \mathrm{M} \mathrm{NaCl}$, pH 7.4) with $0.1 \%$ Tween 20 , membranes were incubated with primary antibodies overnight at $4{ }^{\circ} \mathrm{C}$ and then probed with secondary antibodies conjugated with HRP (DAKO). Primary antibodies used in this study were anti-human UCP1 (Abcam, co, Cambridge, MA, USA), anti-human PPAR $\gamma$, anti-human PGC-1 $\alpha$, anti-human OXPHOS (Abcam, co, Cambridge, MA, USA), and antihuman GAPDH (Cell Signaling Technology, Beverly, MA, USA).

\section{Measurement of mitochondrial copy number}

Total DNAs (genomic and mitochondrial DNA) were isolated from differentiated cells using the QiaAmp DNA Mini kit (Qiagen, Hilden, Germany) according to the manufacturer's instructions. DNA concentration was assessed with a Nanodrop 2000 (Thermo Fisher Scientific). The mtDNA copy number relative to genomic DNA content was quantitatively analyzed by Prism VIIA7 real-time PCR system (Thermo Fisher Scientific). mtND1 and $\beta$-globin primers were as follows:

mtND1: (forward) GTCAACCTCGCTTCCCCACCCT, (reverse) TCCTGCGAATAGGCTTCCGGCT; and $\beta$ globin: (forward) CGACGGGAGGGTCGGGACAA, (reverse) GCCCCGCGAAAGAGCGGAAG.

\section{Oil-Red-O staining}

The neutral lipids of differentiated cells were stained with $0.2 \%$ (w/v) Oil-Red 0 (Sigma-Aldrich, St. Louis, MO, USA) for $10 \mathrm{~min}$ at room temperature after being fixed with $10 \%$ paraformaldehyde. Stained cells were studied using light microscopy (LSM 780, ZEISS, Germany).

\section{Immunocytochemistry}

To assess the expression of brown adipogenic markers, differentiated cells were stained with antihuman UCP1 (1 $\mu \mathrm{g} / \mathrm{mL})$ and then stained with Alexa 488-conjugated secondary antibody (Invitrogen), BODIPY (Thermo), and DAPI (Leagene), described by the manufacturer's instructions. Cells positive for both UCP1 and BODIPY were determined to be brown/beige adipocytes. Cells stained with secondary antibody while omitting the primary antibody served as negative controls. Images were taken using Zeiss laser scanning confocal microscopy (LSM780, Germany).

\section{Measurements of oxygen consumption}

eWAsc and aWAsc were seeded ( $1 \times 10^{4}$ cells/well) into XF24 V7 cell culture microplates (Seahorse Bioscience) and cultured in DMEM with 20\% FBS and antibiotics (100 units/mL of penicillin and 100 $\mu \mathrm{g} / \mathrm{mL}$ of streptomycin) overnight at $37{ }^{\circ} \mathrm{C}$ under an atmosphere of $5 \% \mathrm{CO}_{2}$. After cells were grown to reach $100 \%$ confluence, they were allowed to differentiate for 6 days. aWAC served as the control. The 
$\mathrm{O}_{2}$ consumption was measured by a Seahorse Bioscience XF24 extracellular flux analyzer (XF24, Seahorse Bioscience). To measure OCR after different stimuli, $1 \mu \mathrm{M}$ oligomycin, $1 \mu \mathrm{M}$ carbonyl cyanide-p-trifluorome thoxyphenylhydrazone, and $2 \mu \mathrm{M}$ respiratory chain inhibitor rotenone were successively added to measure OCR independent of oxidative phosphorylation, the maximal respiration, and basal nonmitochondrial respiration rates, respectively.

\section{RNA Extraction and next generation sequencing}

The total RNA of eWAsc and aWAsc was extracted using TRIzol reagents (Invitrogen, USA) and then measured using an Agilent 2200 Bioanalyzer (Agilent, USA) to evaluate quality and quantity. RNAs with RIN $>8.0$ were used to construct the cDNA library and sequencing was subsequently performed with Illumina Hi-Seq 4000 (Illumina, USA).

\section{RNA-Seq analysis}

Raw data was harvested and then filtered with FastQC software and mapped to the RefGenome (GRCh38/p13) with the HISAT2 algorithm [10]. The mapped reads were normalized with HTSeq [11] to calculate the RNA expression levels of each sample. Visualization was achieved with 3D-PCA, correlationplot, and box-plot results.

Differentially expressed gene (DEG) analysis was applied utilizing the DESeq algorithm [12] based on the following criteria: fold change $>2, p$-value $<0.05$, and FDR $<0.05$. GO analysis with the Gene Ontology database and pathway analysis with the KEGG database were applied for functional annotation. Significance, as evaluated by p-values, was defined by the Fisher's exact test [13] and FDR was calculated by the BH test. Based on a hierarchy of the Gene Ontology database, GO-Tree analysis was performed with GO analysis results, and the Pathway-Act-Network was constructed using the relationships and annotation of all DEGs in the KEGG database.

\section{Statistical analysis}

All data were presented as mean \pm SD. Statistical significance was tested using ANOVA or Student's $t$-test, and $p<0.05$ was considered to be significant.

\section{Results}

Morphology and adipocyte progenitors from human fetal subcutaneous white fat depots

To investigate the morphological character and beige adipogenic potential of eWAsc, subcutaneous white fat tissues were isolated from a biopsy of a human embryo (eWAT), as well as the peritoneal omentum region of an adult (aWAT), which served as the reference control. There were significant differences in morphology between eWAT and aWAT (Fig. $1 \mathrm{~A}$ and $1 \mathrm{~B}$ ). eWAT appeared to be a collection of dispersed fat particles that were not yet fully developed, while aWAT was a cluster of expanded mature fat deposits that were filled with lipid droplets (Fig. 1A and 1B). The isolation and preparation of eWAsc and aWAsc has been previously described [8]. In addition, the isolated cells of eWAsc were clearly larger than from aWAsc (Fig. 1A and 1B), which is supported by data from SSC-A and FSC-A in Fluorescence Activated Cell Sorting (FACS; Fig. 1C and 1D).

Markers of adipogenic precursor cells were also measured by FACS, which was defined as $\mathrm{CD}_{29^{+}} / \mathrm{CD}^{-} 1^{-} / \mathrm{CD} 11 \mathrm{~b}^{-} / \mathrm{CD} 4^{+}$expressing cells [14-16]. The percentage of CD29 ${ }^{+} / \mathrm{CD}^{-} 1^{-}$ and $\mathrm{CD} 11 \mathrm{~b}^{-} / \mathrm{CD} 34^{+}$cells was comparable between eWAsc and aWAsc (Fig. 1E and 1F). The percentage of $\mathrm{CD}_{29^{+}}$and/or $\mathrm{CD} 34^{+}$cells was calculated and compared between eWAsc and aWAsc (Fig. 1G and $1 \mathrm{H}$ ). The proportion of adipogenic progenitors in eWAsc and aWAsc, defined as CD29 ${ }^{+} / \mathrm{CD}^{2} 4^{+} / \mathrm{CD} 31^{-} / \mathrm{CD} 11 \mathrm{~b}^{-}$cells, were quantified by FACS (Fig. $1 \mathrm{G}$ and $1 \mathrm{H}$ ). Interestingly, the proportion of typical preadipocytes in aWAsc, defined as CD34 ${ }^{+} / \mathrm{CD}^{-} 1^{-} /$ $\mathrm{CD}_{11 \mathrm{~b}^{-}}$cells was significantly higher than in eWAsc (Fig. 1H). This is could be due to the fact that eWAsc was derived from immature embryonic adipose tissue and would therefore retain earlier progenitor characteristics or pluripotency, while aWAsc was derived from mature adult fat tissue and are more likely to be characteristic of advanced adipose precursor cells. 


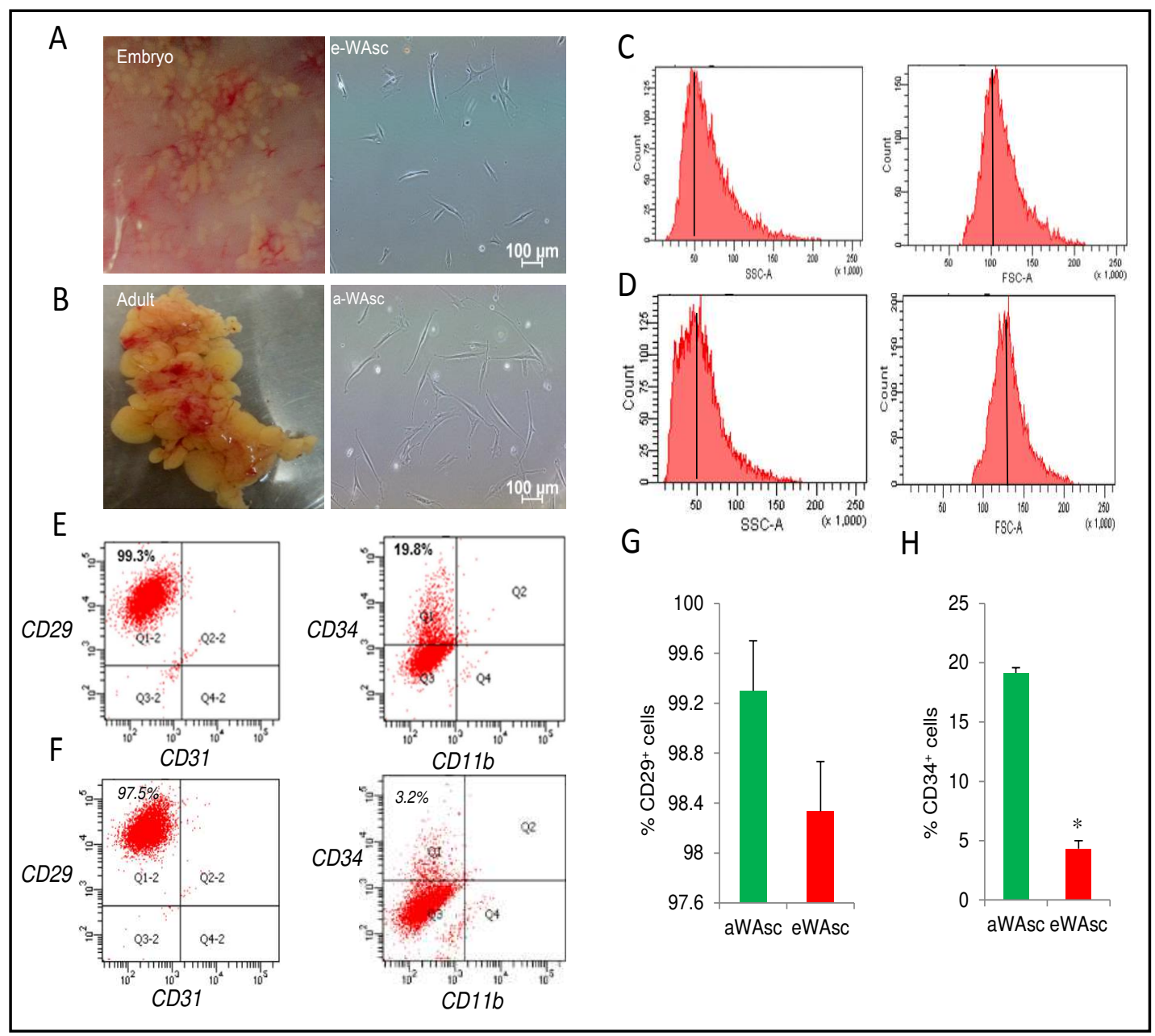

Fig. 1. Morphology and adipocyte progenitors of eWAsc. White adipose tissue was obtained from adults and embryos, while cells from the stromal vascular fraction were isolated. (A) The morphology of white adipose tissue obtained from the legs and back location in human fetus (left) and the separated eWAsc (right) (B) The morphology of white adipose tissue obtained from the abdominal omentum in adults (left) and the separated aWAsc (right). (C) SSA-A and FSC-A of eWAsc analyzed by flow cytometry. (D) SSA-A and FSC-A of aWAsc analyzed by flow cytometry. Both eWAsc and aWAsc were stained with antibodies against CD29, CD34, CD31, and CD11b. Representative FACS dot plot analysis of CD29/CD31 and/or CD34/CD11b of eWAsc and aWAsc are shown in E and F, respectively. E is quoted from our previous study [8]. (G) The percentage of CD29+ cells in eWAsc and aWAsc. $(\mathrm{H})$ The percentage of CD34+ cells in eWAsc and aWAsc. $\mathrm{N}=3$ per group. Differences in expression were considered to be significant if ${ }^{*} \mathrm{p}<0.05$ when compared to aWAsc.

\section{The adipogenic characteristics of eWAsc}

The adipogenicity of isolated eWAsc was next investigated. eWAsc and aWAsc were both induced by induction cocktail for brown adipogenesis and then compared. At 7 days post-differentiation, eWAsc and aWAsc had developed visible lipid droplets as evidenced by phase-contrast appearance and Oil-Red-O staining (Fig. 2A). To evaluate the differentiation ability of eWAsc, the expression of key adipogenic genes-including C/EBP $\alpha, \mathrm{C} / \mathrm{EBP} \beta, \mathrm{C} /$ EBPS, PPARy, PPAR $\alpha$ and AP2-was significantly upregulated in eWAsc compared to aWAsc after induced differentiation (Fig. 2B-2D). The expression of PPAR $\gamma$, a key regulator of adipogenesis both in brown and white adipose tissue $[4,17,18]$, in differentiated eWAsc was further confirmed by immunoblotting (Fig. 2C-2D). These results highlighted that eWAsc has a greater ability/potential for adipogenesis. In addition, we also measured the potential of white adipogenesis for eWAsc and brown adipogenesis for aWAsc. Consistently, key

\section{KARGER}




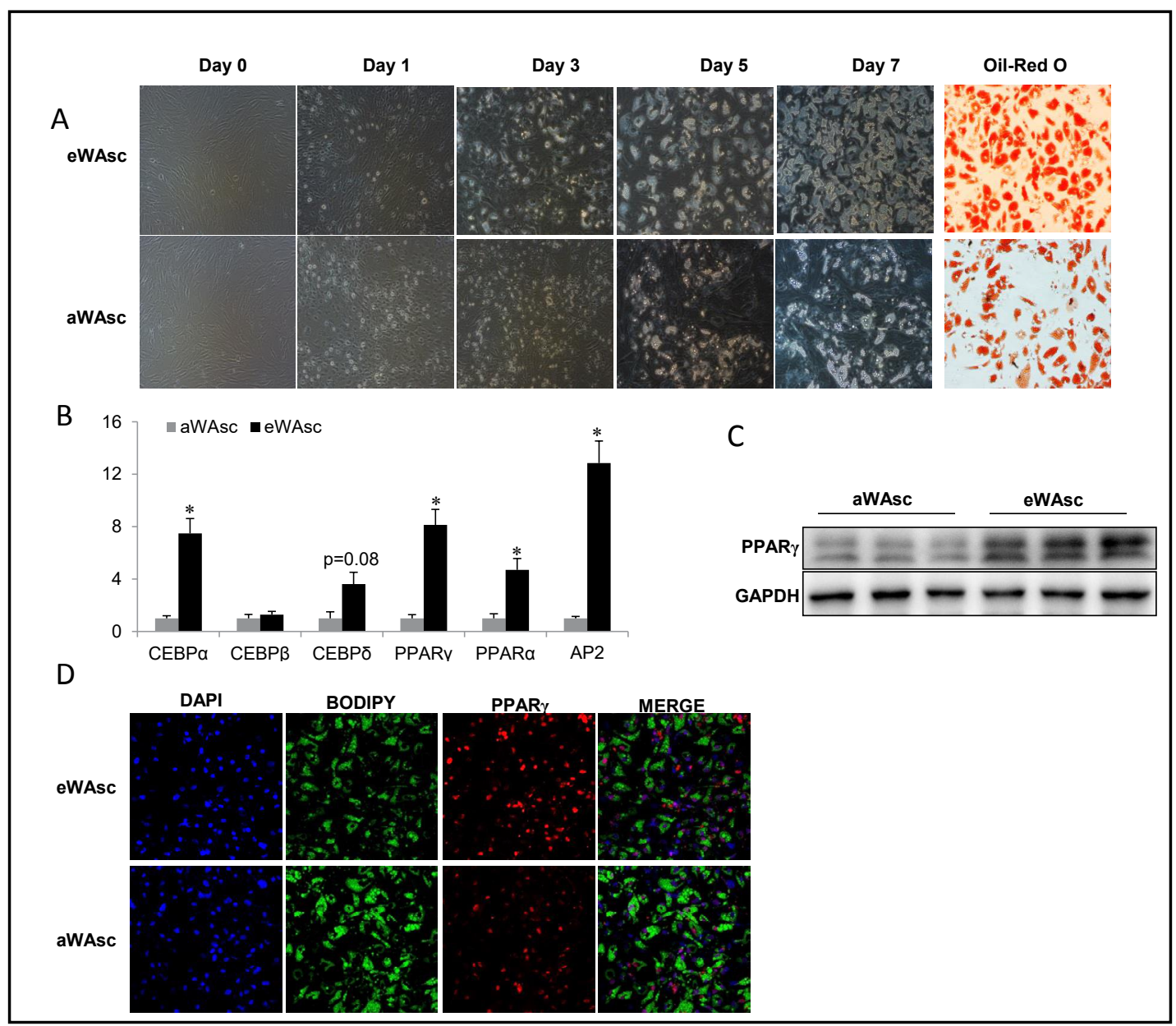

Fig. 2. Characterization of the adipogenic potential of eWAsc. eWAsc and aWAsc were induced with a cocktail to examine brown adipogenesis. (A) Morphology changes during differentiation from Day 0 (without induction cocktail) to Day 7 (after induction cocktail). Mature adipocytes were stained with Oil-

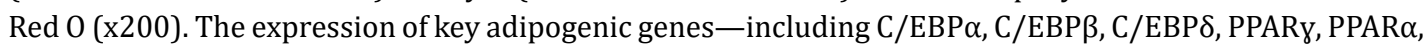
and AP2-in differentiated cells were analyzed by qRT-PCR (B). Data were expressed as fold change. In addition, the expression of PPARy was verified by immunoblotting (C) and immunofluorescence (D). The differentiated eWAsc and aWAsc were stained with anti-human PPARy (red) and BODIPY (green) (x200) (D). $\mathrm{N}=3$ per group. Differences in expression were considered to be significant if $* \mathrm{p}<0.05$ when compared to aWAsc.

adipogenic genes, including $\mathrm{C} / \mathrm{EBP} \alpha / \beta / \delta, \mathrm{ADQ}$, PPARy and AP2, had dramatically increased expression, while the expression of brown adipocytes markers (UCP1, ZIC1, DIO2 and PRDM16) and thermogenic genes (PGC1 $\alpha, \mathrm{PGC} 1 \beta, \mathrm{CPT} 1 \alpha$ and CPT1 $\beta$ ) were not significantly different after white and/or brown adipogenic induction cocktail treatment in eWAsc and/ or aWAsc respectively (Fig. 3 and Fig. 4).

\section{The beiging potential of eWAsc}

We next investigated the potential of eWAsc for beige adipogenesis. Upon induction, UCP1, the best representative marker for brown adipocyte-specific identification, was dramatically increased by Day 7 after induction in comparison to Day 0 , as quantified by quantitative realtime PCR (qRT-PCR) and immunoblotting (Fig. 5A, 5C, and 5D). In addition, the expression of thermogenic genes-such as PRDM16, PGC1 $\alpha$, PGC1 $\beta$, CPT1 $\alpha$ and CPT1 $1 \beta$-was significantly increased at Day 7 compared with Day 0 (Fig. 5A). Furthermore, the expression of beige adipocytes marker genes (CD137, TBX1, and TMEM26) was measured. CD137 and TBX1

\section{KARGER}




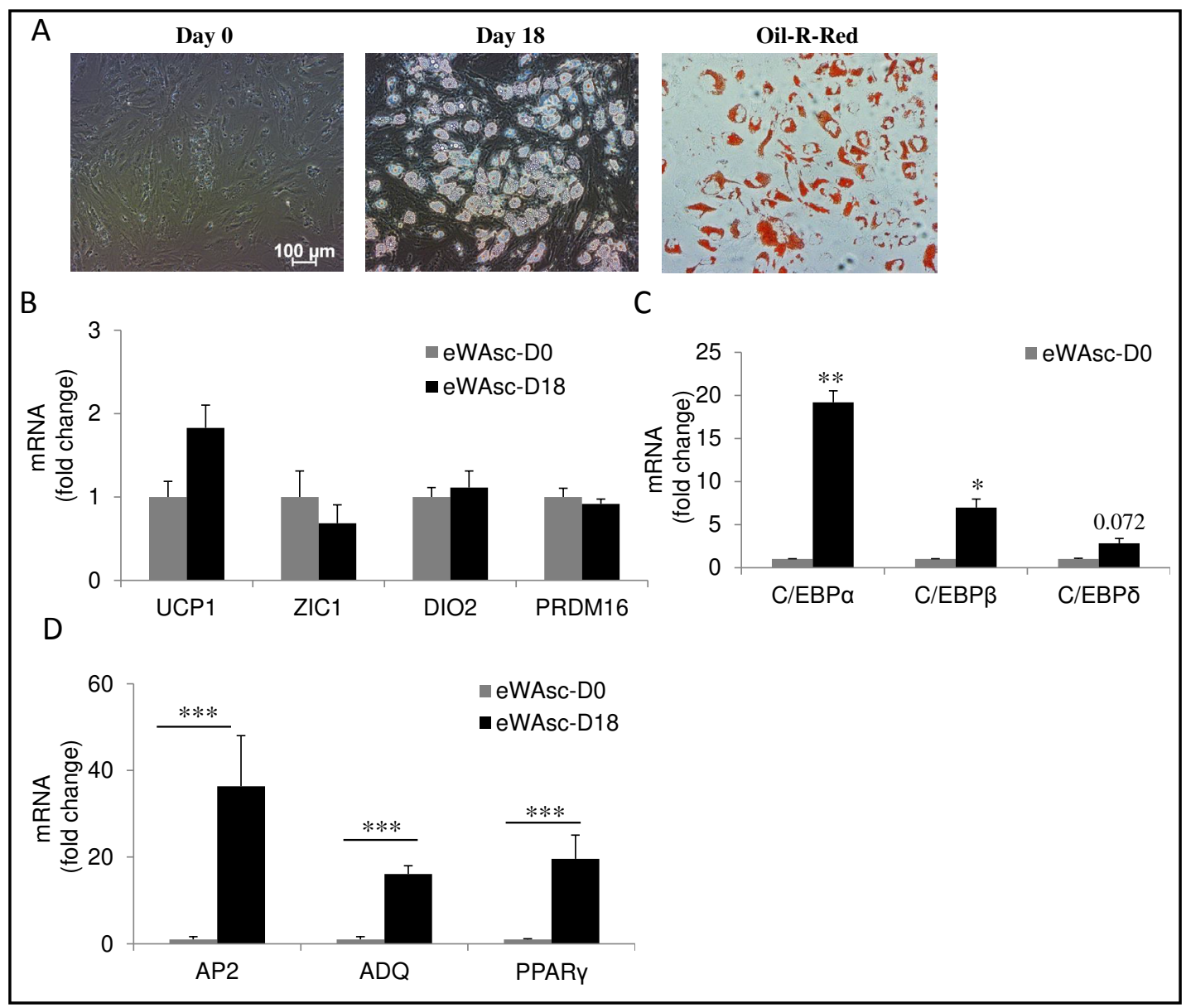

Fig. 3. White adipogenesis of eWAsc. (A) Oil-R-Red was used to examine the morphology of differentiated eWAsc at Day 0 (without induction cocktail) and Day 18 (after addition of induction cocktail for white adipocytes) and of mature adipocytes (x200). The brown adipocytes marker genes-including UCP1, ZIC1, DIO2 and PRDM16-were analyzed by qRT-PCR (B). The expression of key adipogenic genes, including $\mathrm{C} / \mathrm{EBP} \alpha / \beta / \delta, \mathrm{ADQ}$ PPARy, and AP2, in differentiated cells were analyzed by qRT-PCR (C-D). Data were expressed as fold change. $\mathrm{N}=3$ per group. Differences in expression were considered to be significant if ${ }^{*} \mathrm{p}<0.05,{ }^{* *} \mathrm{p}<0.01$ and ${ }^{* * *} \mathrm{p}<0.001$ when compared to Day 0 .

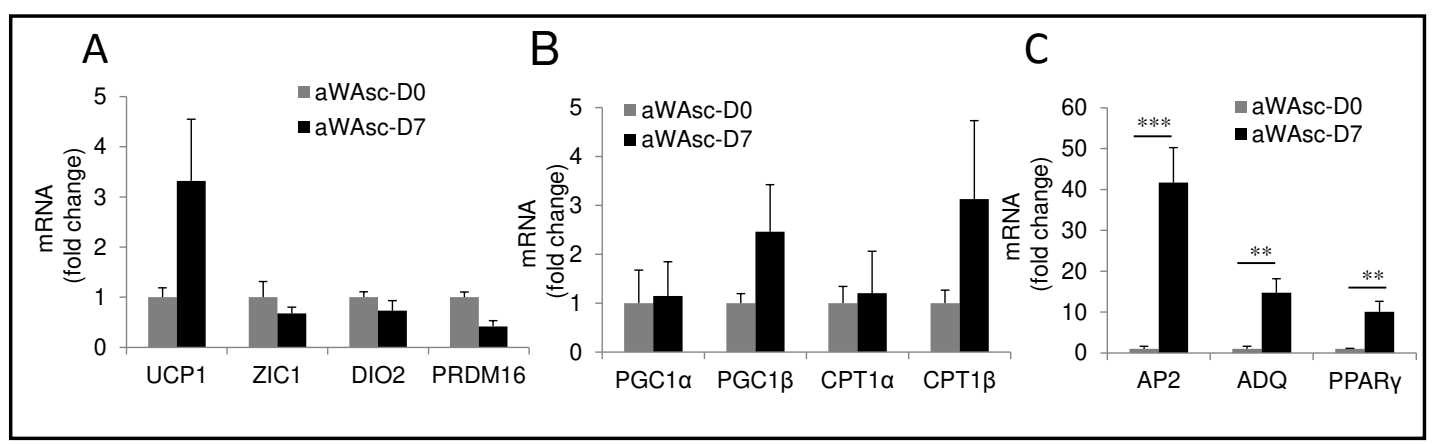

Fig. 4. Brown adipogenesis of aWAsc. The brown adipocytes marker genes-including UCP1, ZIC1, DIO2 and PRDM16-were analyzed by qRT-PCR (A). The expression of thermogenic genes, including PGC1 $\alpha$, PGC1 $\beta$, CPT1 $\alpha$ and CPT1 $\beta$ in differentiated cells, were analyzed by qRT-PCR (B). The key adipogenic genes, including ADQ, PPARy, and AP2 in differentiated cells, were analyzed by qRT-PCR (C). Data were expressed as fold change. $\mathrm{N}=3$ per group. Differences in expression were considered to be significant if $* \mathrm{p}<0.05$, ${ }^{* *} \mathrm{p}<0.01$ and ${ }^{* * *} \mathrm{p}<0.001$ when compared to day 0 . 
Zhang et al.: Beige Adipocytes and Human Embryo White Adipose Tissue-Derived Stem Cells

expression were dramatically increased 7 days after induction in comparison to Day 0 (Fig. 5B). Immunoblotting data further confirmed upregulation of UCP1 and PGC1 $\alpha$, as well as OXPHOS proteins-including ATP5A, ubiquinol-cytochrome C reductase core protein II (UQCRC2), and succinate dehydrogenase complex and subunit B (SDHB) -in eWAsc after 7 days of stimulation (Fig. 5D).

Comparison of the beige adipogenic potential between eWAsc and aWAsc

To further investigate and highlight the beige adipogenic potential of eWAsc, we compared the beige adipogenic potential of eWAsc and aWAsc, defining the differentiated cells as eWAC and aWAC, respectively. Therefore, the expression of BAT marker genes (UCP1, PRDM16 and Z1C1) was analyzed by qRT-PCR after induction of brown adipogenesis. Unsurprisingly, the expression of UCP1 was dramatically increased in eWAC compared to aWAC, but there was no significant difference in ZIC1 and PRDM16 expression ( $p=0.057)$ (Fig. 6A). Additionally, the thermogenic genes-including PGC1 $\alpha, \mathrm{PGC} 1 \beta, \mathrm{CPT} 1 \alpha$ and CPT1 $1 \beta$-were upregulated in eWAC when compared with aWAC (Fig. 6B). Next, beige adipocytes marker gene (CD137, TBX1, and TMEM26) expression was measured, revealing that CD137 and TBX1 expression was dramatically increased, but there was no significantly difference in TEME26 expression (Fig. 6C) when comparing eWAC to aWAC. Expression of BAT function markers was further

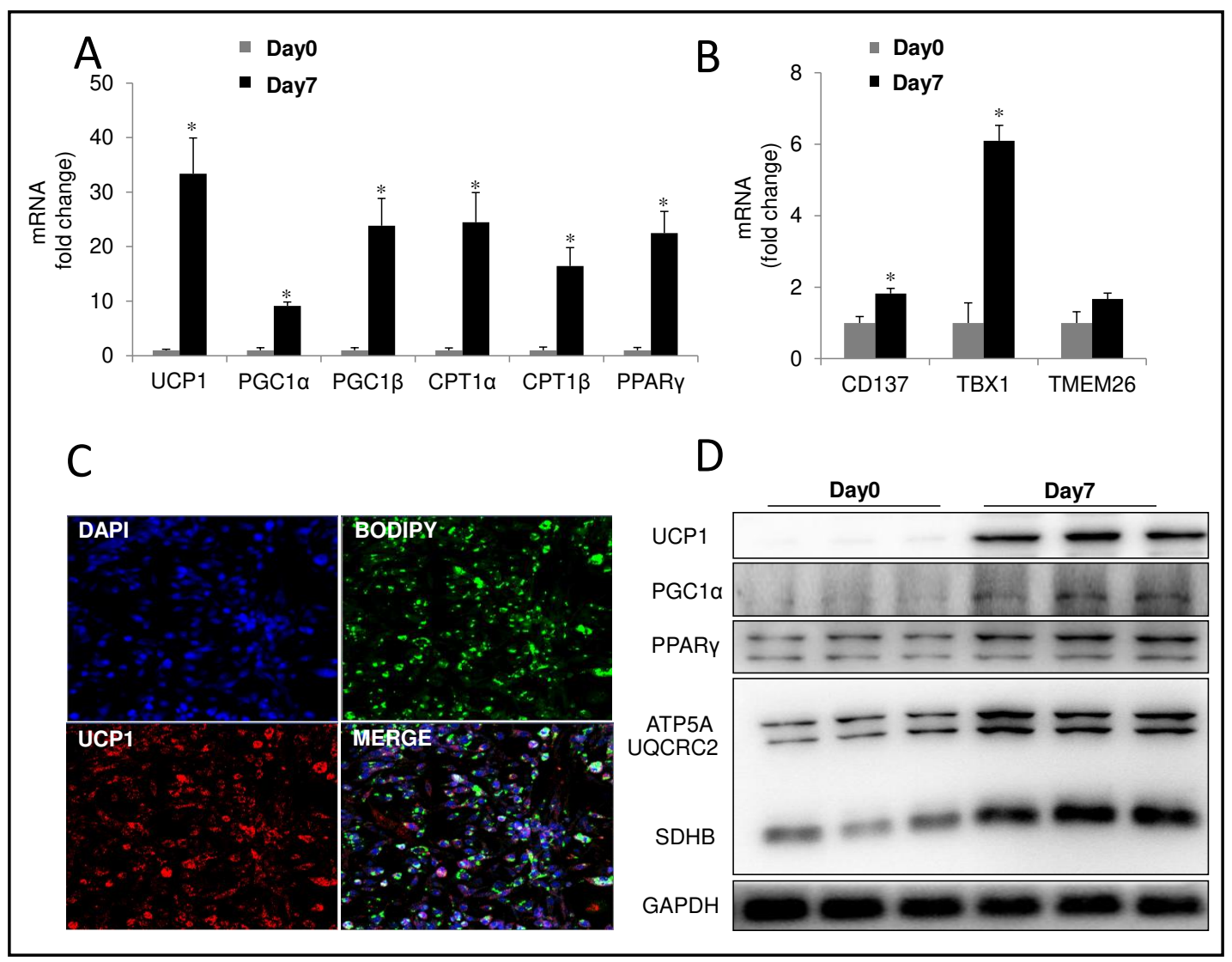

Fig. 5. Beige adipogenesis potential of eWAsc. After inducing differentiation for 7 days, eWAC had increased UCP1 expression at both mRNA (A) and protein (D) levels. In parallel, expression thermogenic genesincluding PGC $1 \alpha$., PGC1 $\beta$, CPT $1 \alpha$, and CPT1 $\beta$-were evaluated by qRT-PCR (A). Expression of beige adipocyte marker genes including-CD137, TBX1, and TMEM26-were analyzed by qRT-PCR (B). In addition, UCP1 expression after induction 7 days (Day7) was verified by immunofluorescence (C). eWAC were stained with anti-human UCP1 (red) and BODIPY (green) (x200) (C). (D) Immunoblot of the expression of UCP1, PGC1 $\alpha$, and OXPHOS (ATP5A, UQCRC2 and SDHB). N=3 per group. Differences in expression were considered to be significant if $* \mathrm{p}<0.05$ when compared to eWAsc (Day0). 


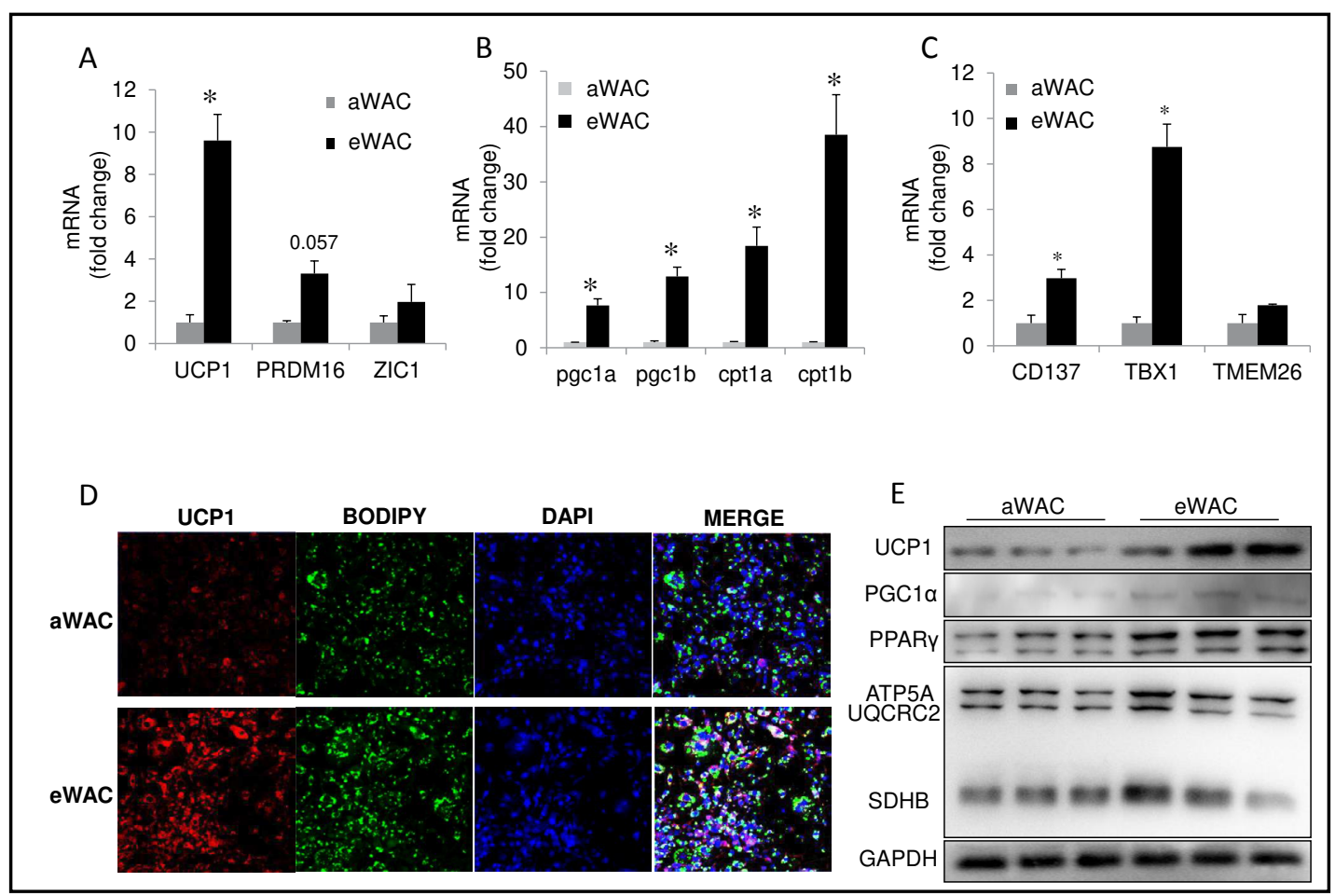

Fig. 6. Comparison of the beige adipogenic potential between eWAC and aWAC. eWAsc and aWAsc differentiation was induced for 7 days utilizing the induction cocktail. At the end of differentiation, eWAsc and aWAsc were characterized via qRT-PCR using brown adipocytes marker genes such as UCP1, PRDM16, and ZIC1 (A), fat acid oxidative related genes, including PGC1 $\alpha$, PGC1 $\beta$, CPT1 $\alpha$ and CPT1 $\beta$ (B), and beige cell markers, including CD137, TBX1 and TMEM26 (C). Data were expressed as fold change. Simultaneously, the expression of UCP1, PGC1 $\alpha$, and OXPHOS (ATP5A, UQCRC2, and SDHB) were analyzed by immunoblotting (D). $\mathrm{N}=3$ per group. Differences in expression were considered to be significant if ${ }^{*} \mathrm{p}<0.05$, when compared to aWAC.

validated by immunoblotting (Fig. 6D and 6E). Expression of UCP1 and PGC1 $\alpha$, as well as mitochondrial-specific OXPHOS proteins (ATP5A, UQCRC2, and SDHB), was significantly increased in eWAC when compared to aWAC (Fig. 6E). However, almost no UCP1 signal was observed in aWAC, while significant UCP1 signal was observed in eWAC, which was then validated by immunostaining (Fig. 6D).

\section{Metabolic and functional characterization of beige adipocytes derived from eWAsc}

Abundant mitochondria and enhanced metabolic activity are the key characteristics of brown and beige adipocytes in comparison to white adipocytes. To evaluate the function of the beige adipocytes differentiated from eWAsc, we quantified the mitochondrial copy number and the expression of mitochondrial biogenesis-related genes, including Tfam and NRF1, by qRT-PCR. The mitochondrial copy number and the expression of Tfam and NRF1 were increased in eWAC compared to aWAC (Fig. 7A and 7B). In addition, norepinephrine (NE) released by the sympathetic nervous system treatment promoted the expression of thermogenic markers UCP1 and PGC1a in eWAC but had limited effect on aWAC (Fig. 7C and 7D), indicating that beige adipocytes induced from eWAsc were responsive to thermogenic stimulation. Collectively, these data indicated that eWAC have beige adipogenic potential.

Finally, the functionality of thermogenesis and oxygen consumption of the beige adipocytes induced from eWAsc needed to be verified, however, these functions are mainly achieved through mitochondrial activity. As measuring oxygen consumption rates (OCR) is the golden indicator for mitochondrial respiration, we examined OCR in beige adipocytes induced from eWAsc and aWAsc using respirometry. Quantitation of OCRs demonstrated 


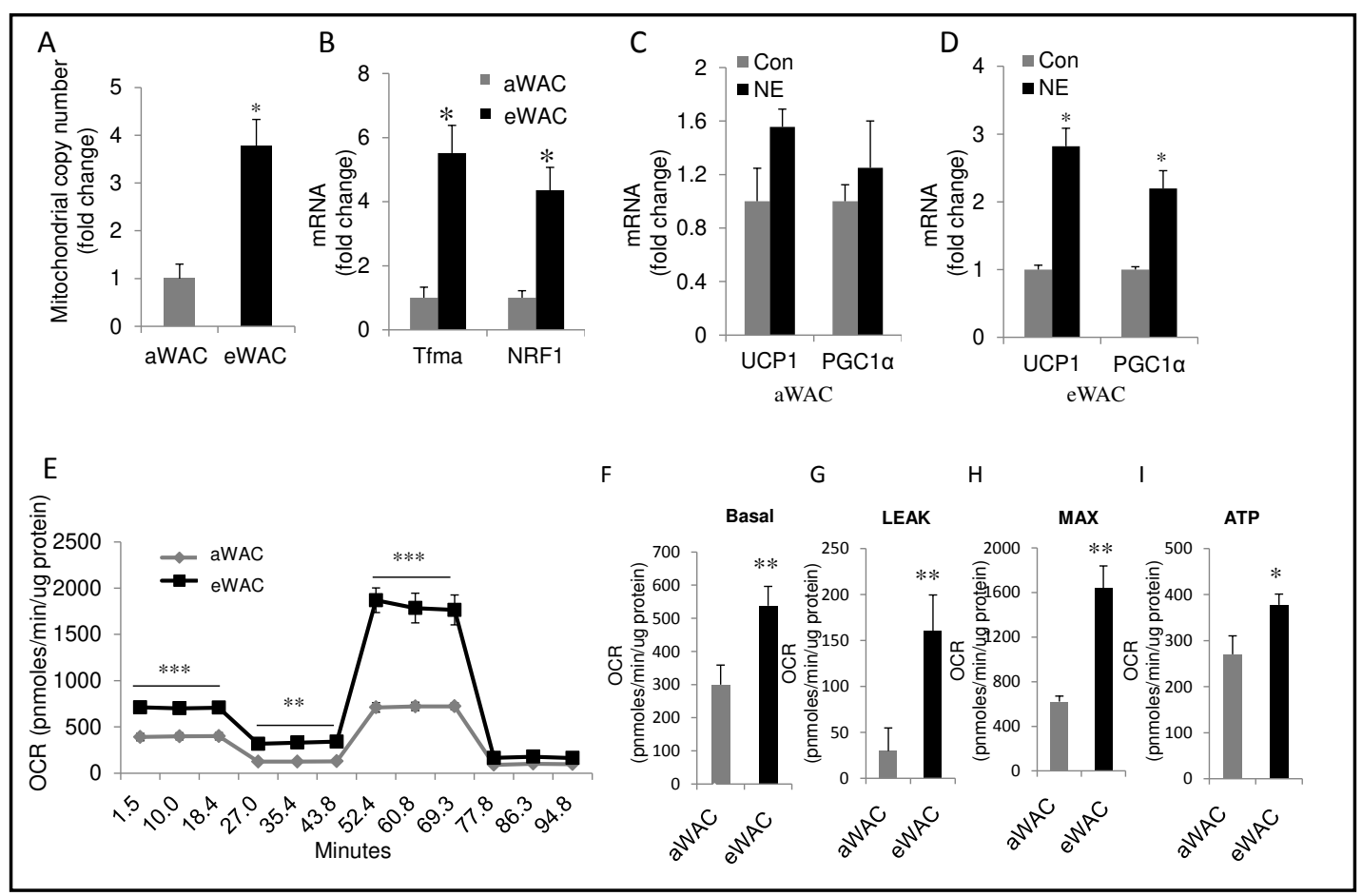

Fig. 7. Mitochondrial genesis and eWAC functionality. To analyze mitochondrial genesis, eWAC and aWAC were analyzed for mitochondrial copy number (A) and mRNA expression of Tfam and NRF1 (B). The effect of norepinephrine (NE) on UCP1 and PGC1 $\alpha$ expression in eWAC and aWAC (C and D). N=3 per group. Differences in expression were considered to be significant if ${ }^{*} \mathrm{p}<0.05$ when compared to aWAC. In addition, to check eWAC functionality, mitochondrial stress experiments were performed to reflect mitochondrial activity. The oxygen consumption rates (OCR) of cells were measured by respirometry (E). Quantification of OCR in Basal, Leak, ATP, and Maximal Respiration (F-I). N=5 per group. Differences in expression were considered to be significant if ${ }^{*} \mathrm{p}<0.05,{ }^{* *} \mathrm{p}<0.01$ and ${ }^{* * *} \mathrm{p}<0.001$ when compared to aWAC.

equal rates of basal respiration and ATP turnover between beige adipocytes induced from eWAsc and aWAsc (Fig. 7E), while the OCR observed from beige adipocytes induced from aWAsc was significantly lower than eWAsc (Fig. 7E). Importantly, beige adipocytes induced from eWAsc had significantly higher rates of maximal respiration, as well as oxygen consumption tied to proton leak, both features consistent with a brown adipocyte metabolic phenotype (Fig. 7F-7I). These data demonstrated that the beige adipocytes induced from eWAsc had excellent metabolic and functionality characteristics.

\section{RNA-Seq analysis of eWAsc and aWAsc}

To further characterize the function, growth regulation, and development of eWAsc, RNAseq analysis was performed on both eWAsc and aWAsc. Significant differentially-expressed genes (DEGs) between eWAsc and aWAsc mice are displayed as a Heat map (Fig. S1A - for all supplementary material see www.karger.com/doi/10.1159/000496042). A volcano map of the data revealed alterations in gene expression in the eWAsc and aWAsc (Fig. S1B). A total of 1263 DEGs were identified between eWAsc and aWAsc, including 605 upregulated and 657 downregulated genes (Fig. S1C). In addition, 137 and 141 genes were specifically expressed in only eWAsc and aWAsc, respectively (Fig. S1D). GO enrichment analysis of DEGs was performed (Fig. S1E) and used to construct a regulatory network of gene function (GO-Tree) to obtain the inherent affiliation of significant function terms (Fig. S1G). According to KEGG databases, pathway-analysis between eWAsc and aWAsc demonstrated that there was high enrichment in cell cycle, TGF- $\beta$ signaling pathway, DNA replication, and the Hippo signaling pathways (Fig. S1F). In order to search the interaction relation between pathway terms, we 


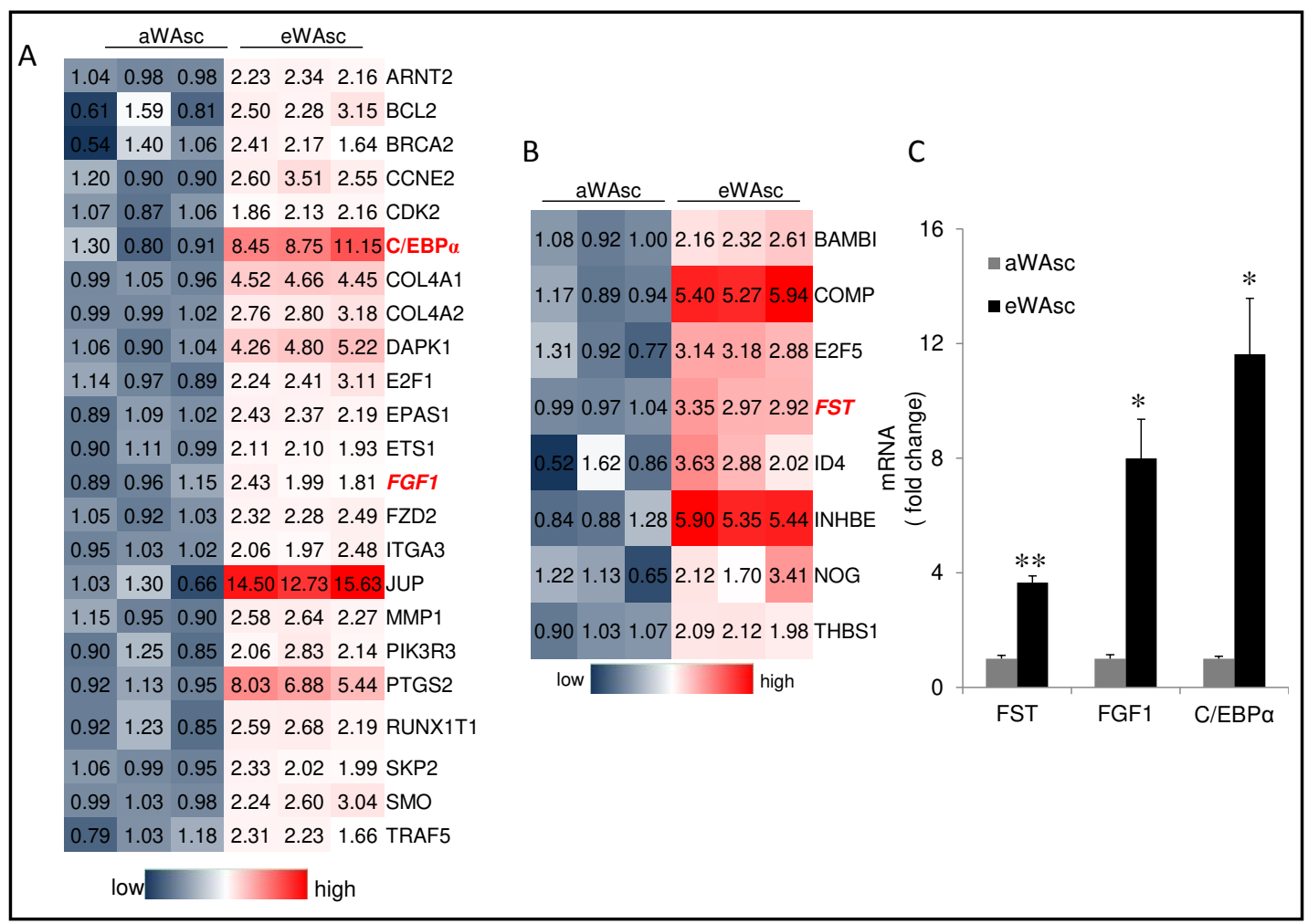

Fig. 8. Heat map of gene validation for of $C / E B P \alpha, F S T$, and FGF1 by RT-qPCR. The heat map of genes associated with cancer pathways (A) and the TGF- $\beta$ signaling pathway (B).C/EBP $\alpha$, FST and FGF1, which are high expression genes in eWAsc, were screened from RNA-seq data and validated by qRT-PCR (C). Differences in expression were considered to be significant if ${ }^{*} \mathrm{p}<0.05$ and ${ }^{* *} \mathrm{p}<0.01$ when compared to aWAsc.

constructed pathway action networks (pathway-act-network) for the significant pathway terms $(P<0.05)($ Fig. S1H). There were cross-talks in multiple pathways, which impacted activation of multiple pathway terms including the cell cycle, TGF- $\beta$ signaling pathway, Wnt signaling pathway, Pathways in cancer, and the Hippo signaling pathways (Fig. S1H).

These analyses revealed that the expression of follistatin (FST), which is associated with the TGF- $\beta$ signaling pathway, and fibroblast growth factor 1 (FGF1), which is associated with cancer pathways and the Hippo signaling pathway, was significantly increased in eWAsc compared to aWAsc (Fig. 8A and 8B). Several studies have indicated that FGF1, an adipokine secreted by adipocytes, is a critical regulator for white and brown adipogenesis [19-23] and that FST, a single-chain gonadal protein that specifically inhibits follicle-stimulating hormone release, can promote adipocyte differentiation and browning/beiging [24-27]. In addition, the expression of C/EBP $\alpha$, which is associated maturation of adipocytes, was also obviously elevated in eWAsc compared to aWAsc (Fig. 8A). Correspondingly, we verified the expression of C/EBP $\alpha$, FST and FGF1 by qRT-PCR (Fig. 8C). These results suggested that the beiging potential of eWAsc may be via activation of the TGF- $\beta$ signaling pathway and Hippo signaling pathway. C/EBP $\alpha$, FST and FGF1 may be potential candidates and possible regulatory targets in recruiting beige adipocytes in human adipose tissue.

\section{Discussion}

In recent decades, the occurrence of metabolic diseases has increased and received greater attention from the medical community $[28,29]$. Therapeutic methods targeting brown adipocytes/beige adipocytes are providing new avenues in therapies for obesity 
and related diseases. Although the characteristics of human adipocytes have been studied [14, 17, 30,31], the research related to human brown or beige adipocytes is not comprehensive, especially in terms of their characteristics, sources, development, functions, and applications. Importantly, compared to brown adipocytes, the ability to generate and isolate beige adipocytes from white adipose tissue is a growing field of research. In this study, subcutaneous white adipose tissue was successfully obtained from human embryos. For the first time, we have analyzed the characteristics, beige adipogenic potential, and functionality of eWAsc.

The morphology of adult adipose tissue is well understood, but little is known regarding the morphology of adipose tissue during the embryonic period (eWAT). This study examined the morphology of eWAT and performed flow cytometry analysis of eWAsc precursor markers. eWAT is a diffuse and flattened fat particle that appears to have immature sprouts of adipose tissue compared to aWAT (Fig. 1A and 1B). Further, adipogenic precursor cell markersdefined as $\mathrm{CD}_{29^{+}} / \mathrm{CD}^{-} 1^{-} / \mathrm{CD} 11 \mathrm{~b}^{-} / \mathrm{CD} 4^{+}$expressing cells-in eWAsc are found at lower levels than in aWAsc (Fig. 1E-1H). This suggests that there may be either more stem cells or an earlier progenitor than preadipocytes in eWAT that have a high level of pluripotency, proliferation, and differentiation activity that contributes to forming mature adipose tissue. Together, this implies that eWAsc may have better plasticity, although whether adipocytes in eWAT could serve as brown/beige adipocytes is yet unclear. Therefore, adipogenesis and browning/beiging potential were examined for eWAC using aWAC as a control group. eWAC was found to better undergo cell differentiation and adipogenesis, which is well demonstrated at both the morphology and molecular levels (Fig. 2). Furthermore, to further evaluate the beiging potential of eWAC, beige adipogenic potential between eWAC and aWAC was compared (Fig. 6). Consistently, the expression of brown adipocytes marker UCP1, thermogenic genes, and beige markers (CD137 and TBX1) were obviously increased in eWAC compared to aWAC (Fig. 6). In short, eWAC performed better than aWAC in terms of adipogenesis and beiging potential.

To explore the molecular characteristics and regulation mechanism in the beige adipogenesis of eWAsc, eWAsc was compared to aWAsc using a comprehensive analysis at the transcriptome level. Signal pathway analysis of DEGs revealed pathways involved in cancer, the TGF- $\beta$ signaling pathway, and the cell cycle, as well as the Hippo signaling pathway, all of which are signaling pathways closely related to cell proliferation, differentiation, and adipogenesis. Importantly, three genes (C/EBP $\alpha$, FST, and FGF1) were identified from the analysis and were more abundant in eWAsc compared to aWAsc. This is an exciting discovery, as some studies have found that FGF1 is highly unregulated in white adipose tissue after a high-fat diet and is regulated by PPAR $\gamma$ [19]. Genetic defects of FGF1 in mice lead to an aggressive diabetic phenotype, a more severe inflammatory response, and aberrant adipocyte size distribution [19-21, 23, 32]. FGF1 can promote adipogenesis [23] and is required for WAT remodeling [20]. The PPAR $\gamma$-FGF1 axis is indispensable for maintaining metabolic homeostasis and insulin sensitization [19]. In addition, FST, a single-chain gonadal protein, can promote adipocyte differentiation and browning/beiging [24-27]. FST can play a regulatory role through the TGF- $\beta$ signaling pathway to regulate multiple biological processes (cell growth and differentiation), as well as the secretion of FSH [33-35]. Genetic defects of FGF1 in mice lead to death shortly after birth [36], which has caused difficulties in understanding its biological role. However, FST does promote muscle growth and mass addition [37-40]. Although how FGF1 and FST mediate adipogenesis in human adipocytes remains unclear, this analysis suggests that FGF1 and FST may be involved in mediating white and brown adipogenesis and has therapeutic potential in recruiting beige adipocytes for obesity or metabolic disorders. As we know, C/EBP $\alpha$ is necessary for activating and maintaining PPAR $\gamma$ expression and conferring insulin sensitivity to mature adipocytes [41]. In conjunction with the above analysis, we hypothesize that the combined effect of C/EBP $\alpha$, FST, and FGF1 may explain the browning/beiging potential of eWAsc compared to aWAsc.

This study has made several important discoveries, but it does have several notable limitations. Although the molecular characteristics and beiging potential of eWAsc were 


\section{Cellular Physiology Cell Physiol Biochem 2018;51:2900-2915 \begin{tabular}{ll|l|l} 
and Biochemistry & Dol: 10.1159/000496042 & Published online: 14 December 2018 The Author(s). Published by S. Karger AG, Basel \\
www.karger.com/cpb
\end{tabular} \\ Zhang et al.: Beige Adipocytes and Human Embryo White Adipose Tissue-Derived Stem Cells}

adequately explored, more detail is required in exploring the regulation mechanism. Similar therapies have been performed in mice by using induced mature human brown/beige adipocytes [30,42]. Although we have demonstrated that eWAsc has excellent browning potential and functionality, these human embryo-derived stem cells or adipocytes are still difficult to apply in clinical practice due to ethical and technical limitations. Currently, very few countries allow for stem cells derived from aborted fetuses to be used in clinical treatments, yet they are still widely applicable in exploring the basic fundamentals of human adipocytes. Currently, adipose-derived stem cells from adult humans have been used in various clinical treatments, but we are still a long way from applying human embryonicderived stem cells. From this study, eWAsc was hypothesized to have superior performance in plasticity and functionality over human adult adipose-derived stem cells, which indicates that more research is needed to fully understand the characteristics of human embryonic adipose-derived stem cells.

\section{Conclusion}

In summary, we have described the beige adipogenic potential of primary white adipocytes from human fetal subcutaneous white fat depots. For the first time, we have successfully demonstrated a well-characterized beige adipocyte phenotype, providing a new reference for recruiting brown or beige adipocytes, which have potential research value for studying human adipocytes.

\section{Acknowledgements}

The current research was supported by the National Natural Science Foundation of China (81700684 to CZhang). The authors gratefully acknowledge the technical assistance of Chao Wang (NovelBio. Ltd), Qibing Dong (GeekGeen. Ltd) and Mengyao Li (Beijing Qinglian Biotech Co., Ltd) for RNA-Seq experiment.

\section{Disclosure Statement}

The authors declare no conflicts of interest.

\section{References}

$>1$ B. Cannon, J. Nedergaard: Brown adipose tissue: function and physiological significance. Physiol Rev 2004;84:277-359.

-2 C.H. Saely, K. Geiger, H. Drexel: Brown versus white adipose tissue: a mini-review. Gerontology 2012;58:1523.

-3 B.B. Lowell, B.M. Spiegelman: Towards a molecular understanding of adaptive thermogenesis. Nature 2000;404:652-660.

-4 P. Seale, B. Bjork, W. Yang, S. Kajimura, S. Chin, S. Kuang, A. Scime, S. Devarakonda, H.M. Conroe, H. Erdjument-Bromage, P. Tempst, M.A. Rudnicki, D.R. Beier, B.M. Spiegelman: PRDM16 controls a brown fat/ skeletal muscle switch. Nature 2008;454:961-967.

$>5$ J. Wu, P. Bostrom, L.M. Sparks, L. Ye, J.H. Choi, A.H. Giang, M. Khandekar, K.A. Virtanen, P. Nuutila, G. Schaart, K. Huang, H. Tu, W.D. van Marken Lichtenbelt, J. Hoeks, S. Enerback, P. Schrauwen, B.M. Spiegelman: Beige adipocytes are a distinct type of thermogenic fat cell in mouse and human. Cell 2012;150:366-376.

-6 P. Hossain, B. Kawar, M. El Nahas: Obesity and diabetes in the developing world--a growing challenge. New Eng J Med 2007;356:213-215.

-7 D.W. Haslam, W.P. James: Obesity. Lancet 2005;366:1197-1209. 


\section{Cellular Physiology Cell Physiol Biochem 2018;51:2900-2915 and Biochemistry DOI: 10.1159/000496042 2018 O 2018 The Author(s). Published by S. Karger AG, Basel and Biochemistry $\frac{\text { Published online: } 14 \text { Decenter } 2018 \text { whw.karger.com/cpb }}{\text { Zhang et al.: Beige Adipocytes and Human Embryo White Adipose Tissue-Derived Stem }}$}

Cells

-8 N.N. Wu, C.H. Zhang, H.J. Lee, Y. Ma, X. Wang, X.J. Ma, W. Ma, D. Zhao, Y.M. Feng: Brown adipogenic potential of brown adipocytes and peri-renal adipocytes from human embryo. Sci Rep 2016;6:39193.

-9 P.A. Svensson, K. Lindberg, J.M. Hoffmann, M. Taube, M.J. Pereira, T. Mohsen-Kanson, A.L. Hafner, M. Rizell, J. Palming, C. Dani, M.K. Svensson: Characterization of brown adipose tissue in the human perirenal depot. Obesity 2014;22:1830-1837.

10 D. Kim, B. Langmead, S.L. Salzberg: HISAT: a fast spliced aligner with low memory requirements. Nat Methods 2015;12:357-360.

-11 S. Anders, P.T. Pyl, W. Huber: HTSeq--a Python framework to work with high-throughput sequencing data. Bioinformatics 2015;31:166-169.

12 S. Anders, W. Huber: Differential expression analysis for sequence count data. Genome Biol 2010;11:R106.

13 S. Draghici, P. Khatri, A.L. Tarca, K. Amin, A. Done, C. Voichita, C. Georgescu, R. Romero: A systems biology approach for pathway level analysis. Genome Res 2007;17:1537-1545.

14 R. Xue, M.D. Lynes, J.M. Dreyfuss, F. Shamsi, T.J. Schulz, H. Zhang, T.L. Huang, K.L. Townsend, Y. Li, H. Takahashi, L.S. Weiner, A.P. White, M.S. Lynes, L.L. Rubin, L.J. Goodyear, A.M. Cypess, Y.H. Tseng: Clonal analyses and gene profiling identify genetic biomarkers of the thermogenic potential of human brown and white preadipocytes. Nat Med 2015:21:760-768.

15 G.J. Hausman, M.V. Dodson: Stromal Vascular Cells and Adipogenesis: Cells within Adipose Depots Regulate Adipogenesis. J Genomics 2013;1:56-66.

16 C. Sengenes, A. Miranville, M. Maumus, S. de Barros, R. Busse, A. Bouloumie: Chemotaxis and differentiation of human adipose tissue CD34+/CD31- progenitor cells: role of stromal derived factor-1 released by adipose tissue capillary endothelial cells. Stem cells 2007;25:2269-2276.

17 M. Harms, P. Seale: Brown and beige fat: development, function and therapeutic potential. Nat Med 2013;19:1252-1263.

18 H. Ohno, K. Shinoda, B.M. Spiegelman, S. Kajimura: PPARgamma agonists induce a white-to-brown fat conversion through stabilization of PRDM16 protein. Cell Metabol 2012;15:395-404.

-19 H. Ohta, N. Itoh: Roles of FGFs as Adipokines in Adipose Tissue Development, Remodeling, and Metabolism. Front Endocrinology 2014;5:18.

$\checkmark 20$ J.W. Jonker, J.M. Suh, A.R. Atkins, M. Ahmadian, P. Li, J. Whyte, M. He, H. Juguilon, Y.Q. Yin, C.T. Phillips, R.T. Yu, J.M. Olefsky, R.R. Henry, M. Downes, R.M. Evans: A PPARgamma-FGF1 axis is required for adaptive adipose remodelling and metabolic homeostasis. Nature 2012;485:391-394.

-21 Y. Choi, S. Jang, M.S. Choi, Z.Y. Ryoo, T. Park: Increased expression of FGF1-mediated signaling molecules in adipose tissue of obese mice. J Physiol Biochem 2016;72:157-167.

22 A. Grefhorst, J.C. van den Beukel, E.L. van Houten, J. Steenbergen, J.A. Visser, A.P. Themmen: Estrogens increase expression of bone morphogenetic protein $8 \mathrm{~b}$ in brown adipose tissue of mice. Biol Sex Differ 2015;6:7.

-23 J. He, D.L. Chen, D. Samocha-Bonet, K.R. Gillinder, J.L. Barclay, G.W. Magor, A.C. Perkins, J.R. Greenfield, G. Yang, J.P. Whitehead: Fibroblast growth factor-1 (FGF-1) promotes adipogenesis by downregulation of carboxypeptidase A4 (CPA4) - a negative regulator of adipogenesis implicated in the modulation of local and systemic insulin sensitivity. Growth factors 2016;34:210-216.

24 M. Braga, S.T. Reddy, L. Vergnes, S. Pervin, V. Grijalva, D. Stout, J. David, X. Li, V. Tomasian, C.B. Reid, K.C. Norris, S.U. Devaskar, K. Reue, R. Singh: Follistatin promotes adipocyte differentiation, browning, and energy metabolism. J Lip Res 2014;55:375-384.

25 R. Singh, M. Braga, S. Pervin: Regulation of brown adipocyte metabolism by myostatin/follistatin signaling. Front Cell Dev Biol 2014;2:60.

26 R. Singh, M. Braga, S.T. Reddy, S.J. Lee, M. Parveen, V. Grijalva, L. Vergnes, S. Pervin: Follistatin Targets Distinct Pathways To Promote Brown Adipocyte Characteristics in Brown and White Adipose Tissues. Endocrinology 2017;158:1217-1230.

27 R. Singh, S. Pervin, S.J. Lee, A. Kuo, V. Grijalva, J. David, L. Vergnes, S.T. Reddy: Metabolic profiling of follistatin overexpression: a novel therapeutic strategy for metabolic diseases. Diabetes Metab Syndr Obes 2018;11:65-84.

28 S. Gesta, Y.H. Tseng, C.R. Kahn: Developmental origin of fat: tracking obesity to its source. Cell 2007;131:242-256.

29 T. Farhat, R.J. Iannotti, B.G. Simons-Morton: Overweight, obesity, youth, and health-risk behaviors. Am J Prev Med 2010;38:258-267. 


\section{Cellular Physiology Cell Physiol Biochem 2018;51:2900-2915

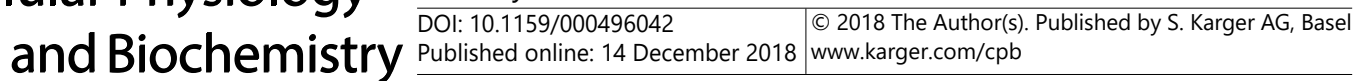 \\ Zhang et al.: Beige Adipocytes and Human Embryo White Adipose Tissue-Derived Stem \\ Cells}

-30 S.Y. Min, J. Kady, M. Nam, R. Rojas-Rodriguez, A. Berkenwald, J.H. Kim, H.L. Noh, J.K. Kim, M.P. Cooper, T. Fitzgibbons, M.A. Brehm, S. Corvera: Human 'brite/beige' adipocytes develop from capillary networks, and their implantation improves metabolic homeostasis in mice. Nat Med2016;22:312-318.

-31 K.M. Tharp, A.K. Jha, J. Kraiczy, A. Yesian, G. Karateev, R. Sinisi, E.A. Dubikovskaya, K.E. Healy, A. Stahl: Matrix-Assisted Transplantation of Functional Beige Adipose Tissue. Diabetes 2015;64:3713-3724.

32 X. Luo, R. Jia, K. Li, X. Zhu, D. Zhao, P.W. J, J. Yan: Fibroblast growth factor-1 inhibits Wnt/beta-catenin pathway during adipogenesis. Journal of Central South University. Medical sciences 2015;40:843-850.

-33 S.J. Lee, Y.S. Lee, T.A. Zimmers, A. Soleimani, M.M. Matzuk, K. Tsuchida, R.D. Cohn, E.R. Barton: Regulation of muscle mass by follistatin and activins. Mol Endocrinol 2010;24:1998-2008.

-34 A.L. Schneyer, Y. Sidis, A. Gulati, J.L. Sun, H. Keutmann, P.A. Krasney: Differential antagonism of activin, myostatin and growth and differentiation factor 11 by wild-type and mutant follistatin. Endocrinology 2008;149:4589-4595.

-35 S.Y. Ying: Inhibins, activins, and follistatins: gonadal proteins modulating the secretion of folliclestimulating hormone. Endocr Rev 1988;9:267-293.

-36 M.M. Matzuk, N. Lu, H. Vogel, K. Sellheyer, D.R. Roop, A. Bradley: Multiple defects and perinatal death in mice deficient in follistatin. Nature 1995;374:360-363.

-37 R. Singh, S. Bhasin, M. Braga, J.N. Artaza, S. Pervin, W.E. Taylor, V. Krishnan, S.K. Sinha, T.B. Rajavashisth, R. Jasuja: Regulation of myogenic differentiation by androgens: cross talk between androgen receptor/ beta-catenin and follistatin/transforming growth factor-beta signaling pathways. Endocrinology 2009;150:1259-1268.

-38 M. Braga, S. Bhasin, R. Jasuja, S. Pervin, R. Singh: Testosterone inhibits transforming growth factor-beta signaling during myogenic differentiation and proliferation of mouse satellite cells: potential role of follistatin in mediating testosterone action. Mol Cell Endocrinol 2012;350:39-52.

-39 C.E. Winbanks, K.L. Weeks, R.E. Thomson, P.V. Sepulveda, C. Beyer, H. Qian, J.L. Chen, J.M. Allen, G.I. Lancaster, M.A. Febbraio, C.A. Harrison, J.R. McMullen, J.S. Chamberlain, P. Gregorevic: Follistatin-mediated skeletal muscle hypertrophy is regulated by Smad3 and mTOR independently of myostatin. J Cell Bio 2012;197:997-1008.

40 H. Gilson, O. Schakman, S. Kalista, P. Lause, K. Tsuchida, J.P. Thissen: Follistatin induces muscle hypertrophy through satellite cell proliferation and inhibition of both myostatin and activin. Am J Physiol Endocrinol Metab 2009;297:E157-164.

41 E.D. Rosen, C.H. Hsu, X. Wang, S. Sakai, M.W. Freeman, F.J. Gonzalez, B.M. Spiegelman: C/EBPalpha induces adipogenesis through PPARgamma: a unified pathway. Genes \& development 2002;16:22-26.

$\checkmark 42$ K.M. Tharp, A. Stahl: Bioengineering Beige Adipose Tissue Therapeutics. Front Endocrinol 2015;6:164. 